\title{
Suture the Feeding and Drainage Vessels to Treat Complicatied Retinal Hemangioblastoma
}

\author{
Hanyi Min ${ }^{1 *}$, Chenxi Zhang ${ }^{1}$, Fei Gao ${ }^{1}$, Rongping Dai ${ }^{1}$, Youxin Chen ${ }^{1}$ and Ningning $\mathrm{Li}^{2}$ \\ ${ }^{1}$ Department of Ophthalmology, Key laboratory of Ocular Fundus Diseases, Peking Union Medical College \\ Hospital, Chinese Academy of Medical Sciences and Peking Union Medical College, Beijing, China \\ ${ }^{2}$ Operating Center, Peking Union Medical College Hospital, Chinese Academy of Medical Sciences and \\ Peking Union Medical College, Beijing, China
}

\section{Introduction}

Retinal hemangioblastoma $(\mathrm{RH})$ is a benign vascular tumor with round, circumscribed appearance, which is often found in the juxtapapillary or peripheral retina. It can be either an isolated vascular abnormality or a manifestation of von Hippel-Lindau syndrome (VHL). RH progresses slowly, but it can lead to significant visual loss from exudative retinal detachment caused by tumor exudation or tractional retinal detachment (TRD) caused by glial proliferation.

In the treatment for $\mathrm{RH}$, peripheral, asymptomatic isolated $\mathrm{RH}$ lesions can be monitored with regular follow-up, while laser photocoagulation and cryotherapy can be used for progressive $\mathrm{RH}$. For larger or more threatening $\mathrm{RH}$ lesions, photodynamic therapy, anti-VEGF treatment, radiotherapy or surgical excision have been proposed to manage.

Vitreoretinal surgery is usually performed for $\mathrm{RHs}>5 \mathrm{~mm}$ in diameter or those complicated by exudative or tractional retinal detachment. $\mathrm{RH}$ excision with or without prior feeder vessel ligation is the most common modality, which achieved good outcome in peripheral, uncomplicated RHs. However, for large $\mathrm{RH}$ located in the posterior pole and complicated by glial proliferation and retinal detachment, tumor excision generally ended in disappointing results with its extensive damage and high risk of hemorrhage. In this study, we report a complicated $\mathrm{RH}$ case in devastating condition managed only with the suture of feeding vessels, which we believe could be an effective treatment modality to control the tumor growth in complicated RHs while minimizing the risk of postoperative complications.

\section{Case Presentation}

A 29-year-old male with known retinal hemangioblastoma $(\mathrm{RH})$ was referred to the Department of Ophthalmology, Peking Union Medical College Hospital for further evaluation. He had been suffered from vision loss due to $\mathrm{RH}$ in his left eye for 10 years, and he received vitrectomy for vitreous hemorrhage caused by RH 10 years ago. After the surgery, the visual acuity in his left eye had been stable with intermittent intravitreal injections of ranibizumab (IVR) and retinal laser photo- coagulation until 9 months ago when retinal detachment and proliferative vitreoretinopathy (PVR) was developed. Vitrectomy was performed again with silicone oil tamponade and postoperative severe hyphema occurred the day after the surgery. After conservative treatment with carbazochrome sodium sulfonate for 3 months, hyphema was absorbed. In the postoperative follow-up, a retinal hemangioblastoma near the inferotemporal vascular arcade in his left eye was found to grow gradually with surrounding shallow retinal detachment, At 9 months postoperatively, retinal detachment was aggravated with extensive peripheral subretinal membrane (SRM) (Figure 1). As to his right eye, the best Snellen corrected visual acuity was 0.8 with normal anterior segment. The retina was attached with localized laser spots due to the asymptomatic multiple retinal hemangiomas applied 10 years ago (Figure 2). Systemic screening showed he had renal hemangioma besides the eyes. The patient was with a family history of von-Hippel Lindau syndrome.

On admission, the best corrected Snellen visual acuity of his silicone-filled left eye was 0.04 with normal intraocular pressure. The central anterior chamber was shallow and the peripheral anterior chamber even disappeared. The posterior capsule opacification was heavy. On the fundus, the RH about $1 \mathrm{PD}$ in size around the inferotemporal vascular arcade was observed with its feeding vessels extending directly from the optic disc to the tumor. The feeding vessels were accompanied by proliferative fibrous cord and the $\mathrm{RH}$ was surrounded by retinal detachment. In the peripheral retina, extensive SRM and focal epiretinal membrane were found and resulted in shallow peripheral retinal detachment. Scattered laser

*Corresponding author: Hanyi Min, Department of Ophthalmology, Peking Union Medical College Hospital, Shuai Fu Yuan, Dongcheng District, Beijing 100730, China, Tel: 086(010)69156365

Accepted: October 22, 2020

Published online: October 24, 2020

Citation: Min H, Zhang C, Gao F, et al. (2020) Suture the Feeding and Drainage Vessels to Treat Complicatied Retinal Hemangioblastoma. J Ophthalmic Surg 3(1):21-24 
Citation: Min H, Zhang C, Gao F, et al. (2020) Suture the Feeding and Drainage Vessels to Treat Complicatied Retinal Hemangioblastoma. J Ophthalmic Surg 3(1):21-24

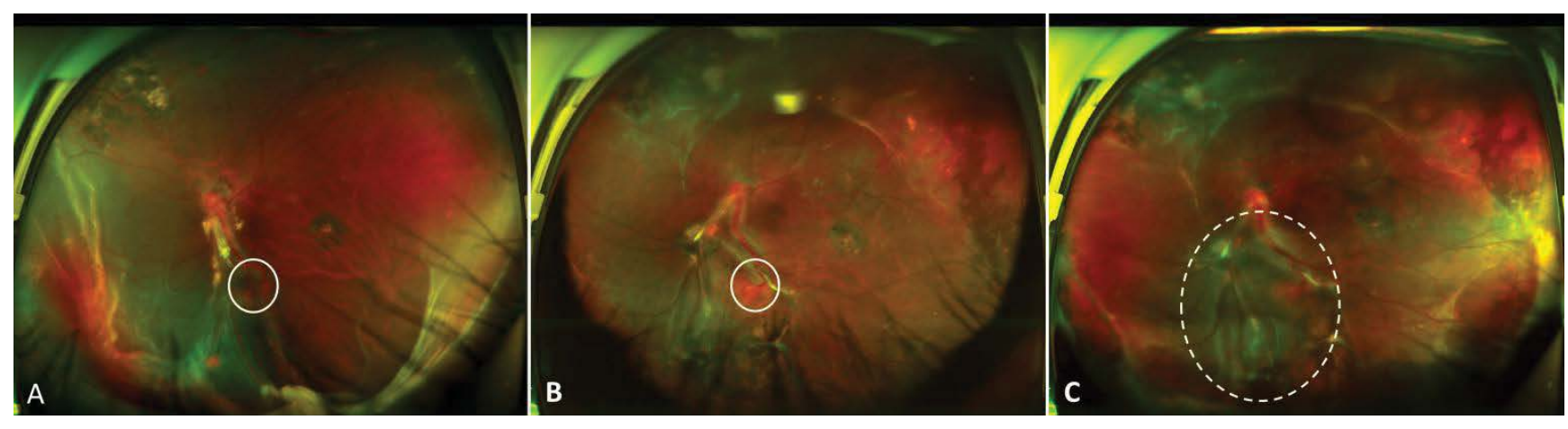

Figure 1: The progression of retinal hemagioma.

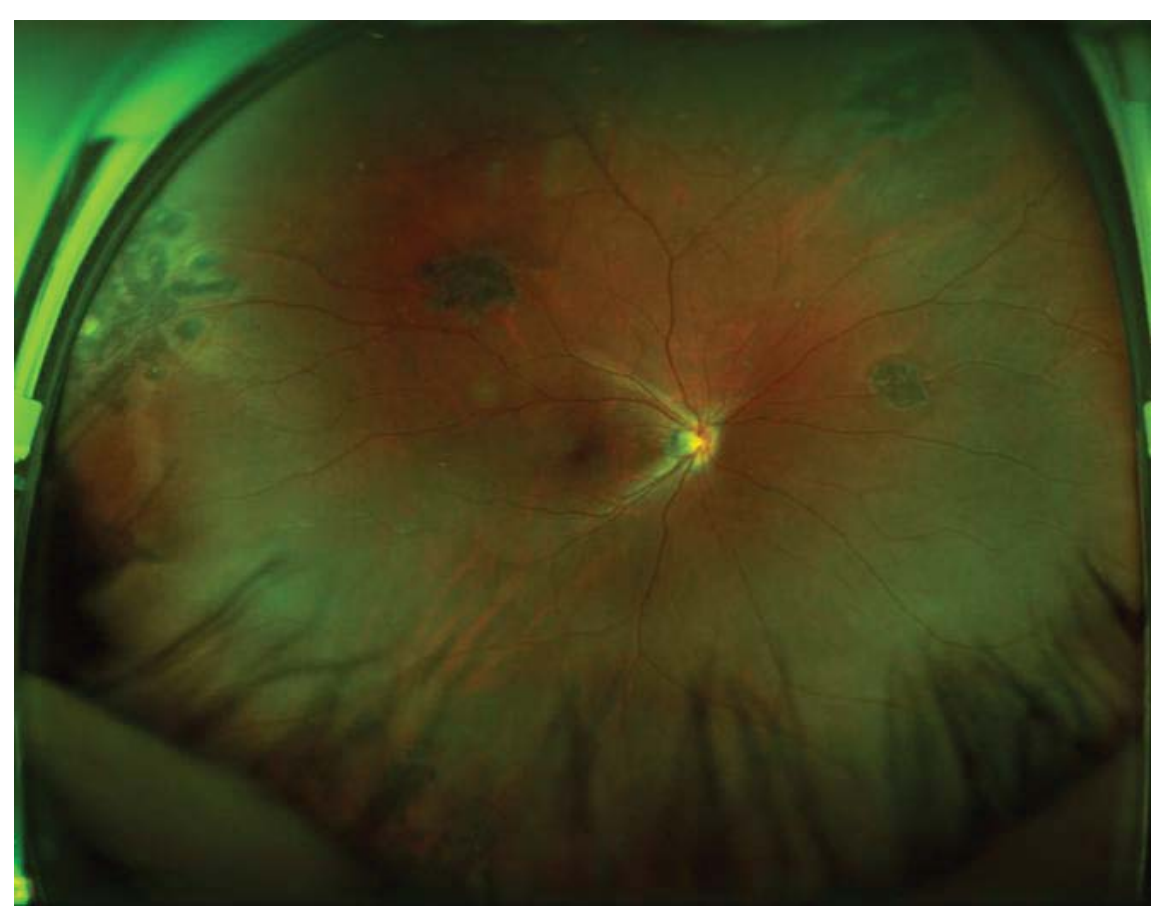

Figure 2: The silent foci of retinal hemagiomas after laser treatment.

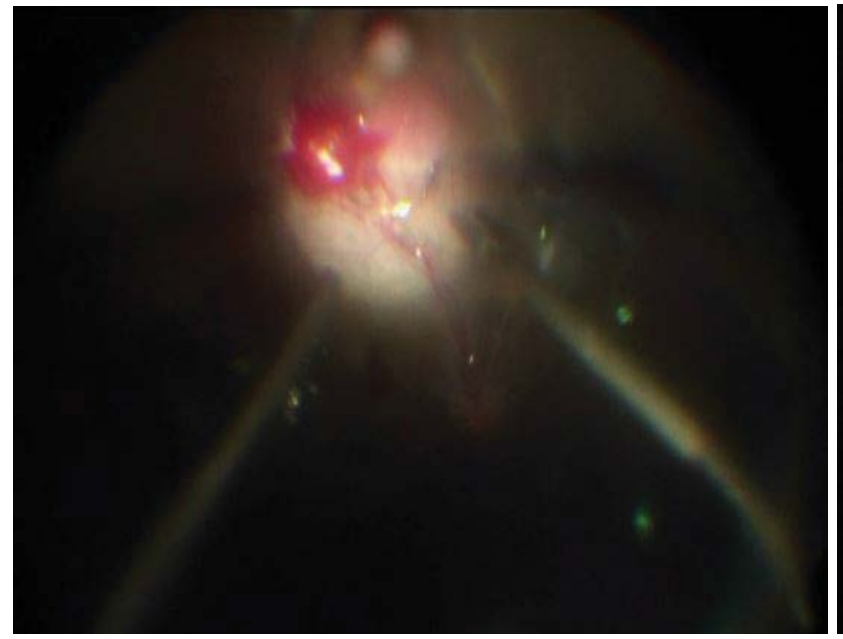

A

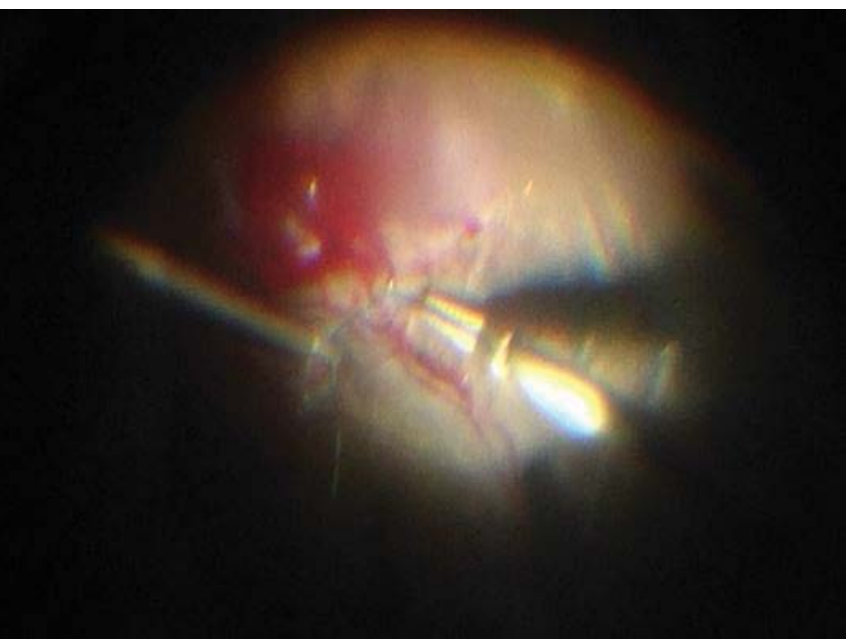

B

Figure 3: Intraoperative procedures (A) The needle applied beneath the vessels; (B) First knot were done tightly and the interrupted blood flow could be observed. 

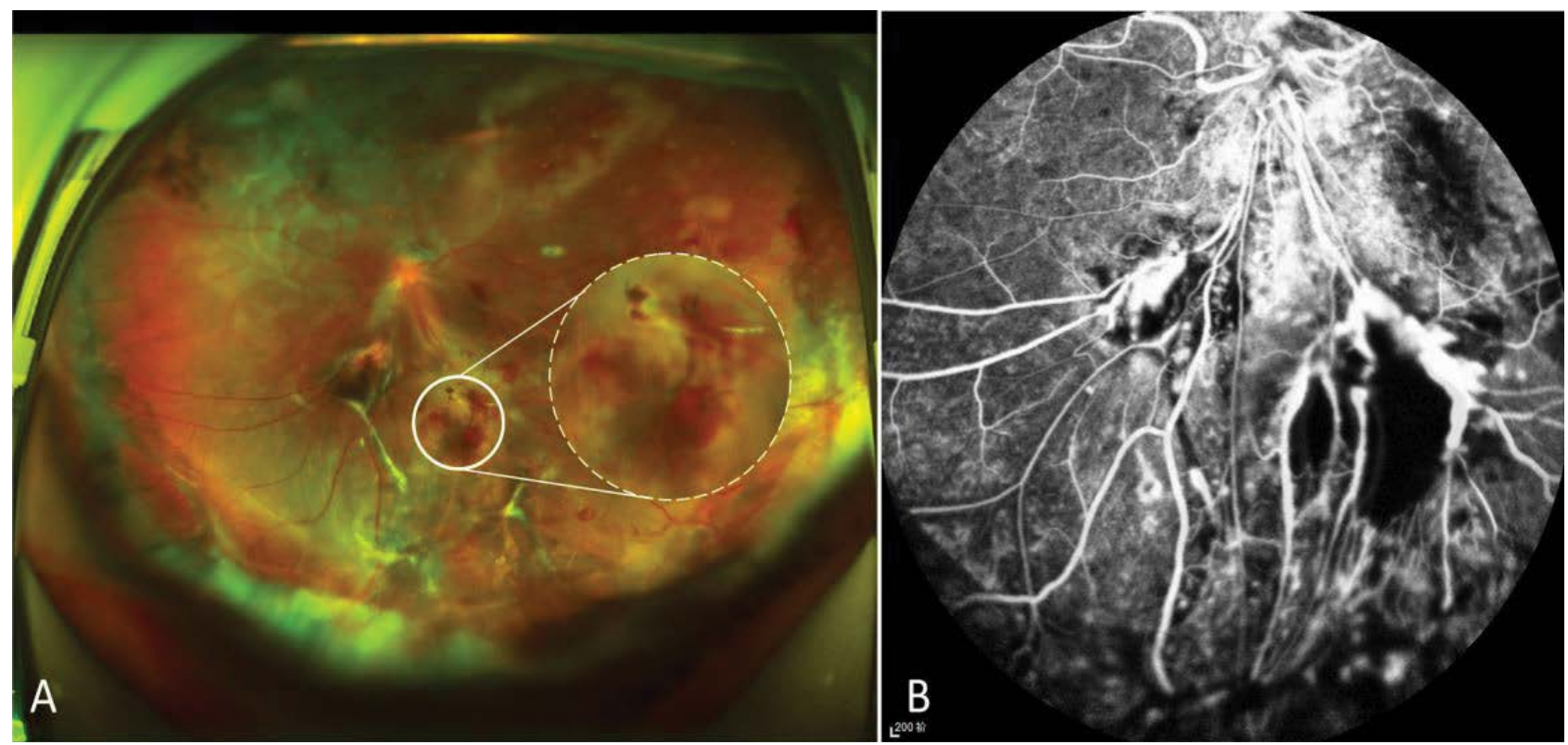

Figure 4: (A) Showed the retina was attached; (B) The blood flow was blocked completely.

spots with pigment proliferation were detected in mid-peripheral retina and temporal macula. Under local anesthesia, we performed a 23-gauge vitrectomy to suture the feeding vessels of $\mathrm{RH}$ in his left eye. The surgery began with anterior chamber plasty, phacoemulsification and posterior capsulotomy. After removal of the silicone oil, we tried to peel the epiretinal membrane around the $\mathrm{RH}$ when massive hemorrhage happened. Deuteroxide was injected and the perfusion pressure was raised to $50 \mathrm{mmHg}$ for a short time to block the bleeding. To suture the $\mathrm{RH}$ feeding and drainage vessels, a 10-0 non-absorbable polypropylene suture was sent into the vitreous cavity through the corneal limbal incision, sutured from one side of feeding vessels and pulled out the stitch out from the other side of the drainage vessel, then tied and ligated the two vessels together genltely and tightly by three konts (Figure 3). The blood flow of the both vessesl was interrupted immediately. The stitch and residual thread was taken out of the eye through the limbal incision. After suturing the feeding vessels, we further dissected peripheral SRM and applied endolaser photocoagulation to the perilesional area. The surgery ended with silicone oil tamponade.

3 days after the surgery, the fundus examination revealed that the suture of the $\mathrm{RH}$ feeding vessels was in place and the retina around the lesion basically reattached. No visualization of the tumor was found in fluorescein angiography, indicating the effectiveness of suture treatment (Figure 4). In the 6-month follow-up, the best corrected Snellen visual acuity of the left eye was 0.05 , the size of $\mathrm{RH}$ was significantly reduced with the suture in place and surrounding retina reattached.

\section{Discussion}

In this study, we explored the possibility of using feeding vessels suture technique in a $\mathrm{RH}$ case with multiple complications and investigated the short-term effectiveness. In this case, the main $\mathrm{RH}$ tumor was with high risk of operation: Posterior pole location, continuing growth and large feeding ves- sels sending directly from the optic disc. And the vitreoretinal condition was complicated: silicone oil filled after repeated vitrectomy, PVR, extensive peripheral subretinal membrane, complicated cataract and shallow anterior chamber. To stop the tumor growth with the least operation, we performed the suture of the feeding vessels of the $\mathrm{RH}$ without tumor excision, which effectively blocking the blood supply, avoiding the damage caused by traditional excision surgery and decreasing the risk of operative complications. The short-term effect was significant that the tumor was significantly reduced and the retina was reattached after 3-month follow-up, visual acuity was maintained stable.

In previous study, van Overdam, et al. reported 4 cases of peripheral retinal hemangioblastoma that underwent early $23 \mathrm{G}$ vitrectomy, of which 2 cases were VHL related. One case received ligation of feeding vessels 17 months before tumor excision. The visual acuities of the 4 cases were stable or improved after operation. 2 cases had mild vitreous hemorrhage postoperatively. Compared with this study, our case is more complicated. The patient had multiple $\mathrm{RHs}$ complicated by PVR, TRD, and SRM after repeated vitrectomy in the left eye. Meanwhile, the main tumor was located in the posterior pole with large feeding vessels, which suggested the probable irresponsibility to anti-VEGF therapy or laser photocoagulation. Massive hemorrhage had been the major concern if to perform tumor excision or vessel dissection before ligation, especially in tumor with severe proliferative membrane. Different from previously reported feeding vessel ligation, the suture of feeding vessels didn't dissect vessel before ligation, therefore decreasing the risk of intraoperative massive hemorrhage, and with enhanced laser photocoagulation, the suture didn't increase the risk of postoperative retinal detachment. Since feeding vessels and surrounding neuroretina are sutured together, the strength needs to be properly handled. If it is too tight, it may still tear the retina and vessels, leading to massive bleeding. If it is too loose to completely cut off 
the blood flow, recanalization will lead to tumor recurrence. Anyway, this technique provides a beneficial attempt for the treatment of complicated RHs in the posterior pole and those with poor responses to laser, anti-VEGF therapy or cryotherapy, but the long-term effect needs further follow-up.
In addition, it is worth noting the patient had bilateral multiple RH lesions and family history of VHL syndrome. The diagnosis of VHL syndrome should be considered even so far no other systemic abnormalities have not been revealed. The general condition of the patient should be regularly followed up, and gene test should be performed if necessary.

DOI: $10.36959 / 587 / 592$

Copyright: (C) $2020 \mathrm{Min} \mathrm{H}$, et al. This is an open-access article distributed under the terms of the Creative Commons Attribution License, which permits unrestricted use, distribution, and reproduction in any medium, provided the original author and source are credited. 